\title{
Low Dispersion Performance of Plastic Fiber Grating Using Genetic Algorithms
}

\author{
Hisham K. Hisham \\ Electrical Engineering Department, Faculty of Engineering, Basra University, Basra, Iraq \\ E-mail: Husham_kadhum@yahoo.com \\ Received: 15-May-2017 Revised: 24-Oct.-2017 Accepted: 18-Dec.-2017 \\ http://doi.org/10.29194/NJES21010045
}

\begin{abstract}
In this paper, we suppose a method for reducing the dispersion in the plastic optical fiber (POF) Bragg gratings based on optimizing the grating coupling-strength $(\xi)$ using genetic algorithms. The effects of average refractive index $(\delta n)$ and temperature $(T)$ change on the dispersion properties are investigated numerically. It is found that the amplitude of the $\delta n$ for low dispersion performance needs to be reduced at the cost of the design complexity of the POF Bragg gratings. Owing to the unusually large and negative thermo-optic coefficient of the POF, the dispersion due to the wavelength shift induced by the temperature variation will be reduced by operating at high $\xi$ value. Results showed that by optimizing the $\xi$ value a very large dispersion reduction range has been obtained, from 1178 to $11.5 \mathrm{ps} / \mathrm{nm}$ at $30 \mathrm{~mm}$ grating length.
\end{abstract}

Keywords: Dispersion in optical fiber, plastic optical fiber (POF), genetic algorithms.

\section{Introduction}

With the large increase in demand for highspeed data transmission over Internet access, fiber Bragg gratings (FBGs) have been developed to support many applications in optical communication systems [1]. However, due to large Young's modulus and a small thermal expansion coefficient, Bragg gratings based silica optical fiber (SOF) cannot be tuned easily by their mechanical or thermal methods. Typically, SOF gratings can only be tuned within 4-6 nm by temperature and mechanical stress [2]. The case in Bragg gratings based plastic (polymer) optical fiber (POF) is totally different; the Young's modulus for the polymer is more than 70 times smaller $[3,4]$ than that for the silica, that makes the tunability much better than that of SOF Bragg gratings. In addition, POF has the merits of a negative and large thermo-optic effect, thereby, large refractive index tuning by heating can be obtained as compared to SOF [5-6]. Therefore, wide tuning range can be obtained easily by direct heating for POF Bragg gratings.

However, the most important critical limitation that determines the transmission bit-rate over optical fiber is the dispersion of the fiber.
Where, it degrades the performance characteristics of optical communication in high speed and long haul systems [7-10]. Dispersion is the result of the non-linear fiber material's and the distance of the traveling signals inside the fiber. In dispersion, different signals travel with different velocities. Due to which all the signals reach at different times which results in "pulse broadening" [11].

Several experimental and theoretical studies have been reported on reducing the dispersion properties in POF Bragg gratings [7-14]. However, they require complicated structures or special fiber designs. In this paper, we propose a simple method for optimizing POF Bragg gratings design by using genetic algorithms. We demonstrated that in this technique the dispersion properties can be eliminated by optimizing the coupling- strength value for the fiber grating. Then based on the optimal values that are obtained by the genetic algorithms, the effect of average refractive index and temperature variation on the dispersion properties are investigated numerically. The paper is structured as follows: The theory for the fiber Bragg grating in plastic optical fibers is given in Section II. Section III discusses the model for the genetic algorithms. The simulation results are discussed in Section IV followed by the conclusions.

\section{Plastic Grating Fiber Model}

By doping photosensitive materials into POF, the refractive index will change upon exposure to bright light. There are many mechanisms that result in a refractive index change in plastic fibers [14]. Since the operation of Bragg gratings is based on the reflection of light from grating fringes and coupling of the modes, thus the maximum reflectivity is obtained at the wavelength that satisfies the Bragg condition [2, 11]:

By assuming $A_{f}(z)$ and $B_{b}(z)$ (as shown in Fig. 1) are the amplitudes of the forward and backward waves, the propagation through the fiber grating is described by a matrix $H$, which is defined such that [11] 


$$
\left(\begin{array}{l}
A_{f}(z) \exp (-j \delta z) \\
B_{b}(z) \exp (+j \delta z)
\end{array}\right)=H \cdot\left(\begin{array}{l}
A_{f}(0) \\
B_{b}(0)
\end{array}\right)
$$

Where the matrix $H$ for Bragg gratings is given by [11]

$$
\begin{aligned}
H=\left(\begin{array}{rl}
\cosh (\Omega z)-\frac{\delta n}{\Omega} & \sinh (\Omega z) \\
+j \frac{\xi}{\Omega} \sinh (\Omega z) & \\
& -j \frac{\xi}{\Omega} \sinh (\Omega z) \\
& \cosh (\Omega z)+j \frac{\delta n}{\Omega} \sinh (\Omega z)
\end{array}\right)
\end{aligned}
$$

Where, $\delta n$ and $\xi$ is the coupling length for the fiber's grating. $\Omega$ is the grating parameter which is defined by [11]

$$
\Omega=\sqrt{\xi^{2}-\delta n^{2}}
$$

For a uniform fiber gratings of length $L_{g}$ the reflectivity $(\rho)$ can be obtained by assuming a forward- propagating incident from $z=-\infty$ and no backward propagation for $Z \geq L_{g} / 2$.

$\rho=\frac{B_{b}\left(-L_{g} / 2\right)}{A_{f}\left(-L_{g} / 2\right)}=\frac{-\xi \sinh \left(\Omega L_{g}\right)}{\delta n \sinh \left(\Omega L_{g}\right)+j \Omega \cosh \left(\Omega L_{g}\right)}$

Based on (4), the maximum reflectivity for gratings fiber occurs when the Bragg condition is satisfied (i.e. the average refractive index $(\delta n)$ approaches zero).

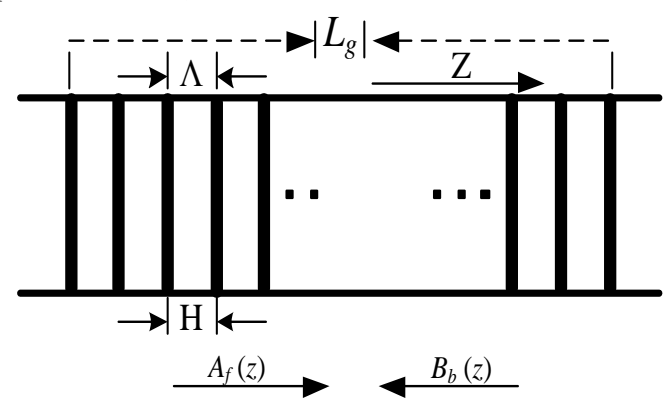

Figure 1: Uniform Bragg gratings in plastic fibers with forward $\left(A_{f}(z)\right)$ and backward $\left(B_{b}(z)\right)$ waves.

\section{Optimization Using A Genetic Algorithm}

Low-coupling strength represents one of the design parameters with respect to well model performance. The dispersion characteristics of the plastic fiber grating has a relation to the coupling strength, amplitude of the induced refractive index perturbation, grating length and the reflectivity, which also have important effect on device's performance. Therefore, the goal is to find the optimal coupling strength that produces the dispersion performance close to the minimum value. The relation of the coupling strength and the dispersion of the grating fibers is so complicated that the genetic algorithm (GA) can be used to select the optimal coupling strength value.

The GA model is used to evolve solutions based on the Darwinism rule. The GA's objective function is the plastic fiber grating dispersion's function, which can be defined by [11]:

$$
D=-\frac{2 \pi c}{\lambda^{2}} \cdot \frac{d^{2} \psi_{\rho}}{d \lambda^{2}}
$$

Where $D$ represent the dispersion parameter of the fiber grating and $\psi_{\rho}$ is the phase of fiber grating reflectivity, respectively. Figure 2 shows the GA's flowchart. A chromosome is a binary of the coupling strength. According to Fig. 2, in the first step, GA will generate randomly initial father chromosomes population (i.e. suitable solutions for the dispersion problem), then step two: by using the dispersion formula as an objective function to find the fitness of individuals of the coupling strength, which is used to select the children population. In the next step, the roulette selection operator is used; the crossover and mutation one-point operators to develop the population by keeping the diversity of the population, and at the same time prevent the preliminary convergence to a local minimum. In step four, use the new generated population for a further run of the algorithm. If the end condition is satisfied, the process will stop and return the best solution in current population. If not, go to step 2 . The process will be repeated many times until the optimization is achieved.

\section{Results and Discussion}

Figure 3 shows the effect of coupling-strength

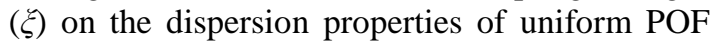
Bragg grating. The dotted line presents the dispersion response with the lowest value that can be calculated by the optimal coupling strength with genetic algorithms. The dashed line presents the dispersion response with the highest dispersion value that can be calculated by the coupling strength with the genetic algorithms. The others curves shown in Fig. 3 are the numerical simulation results with the coupling strength of $2.5 / \mathrm{m}, 10 / \mathrm{m}, 20 / \mathrm{m}, 30 / \mathrm{m}, 50 / \mathrm{m}$ and $60 / \mathrm{m}$, respectively. 
From Fig. 3, it is obvious that the POF Bragg grating with the lowest dispersion response obtained by the genetic algorithms has the highest $\xi$ value of $60 / \mathrm{m}$. Moreover, it is shown that the dispersion response fluctuates within a specified range with the change of the gratings length $\left(L_{g}\right)$. In addition, Fig. 3 shows high consensus between the results that obtained by numerical analysis and that obtained by the genetic algorithms.

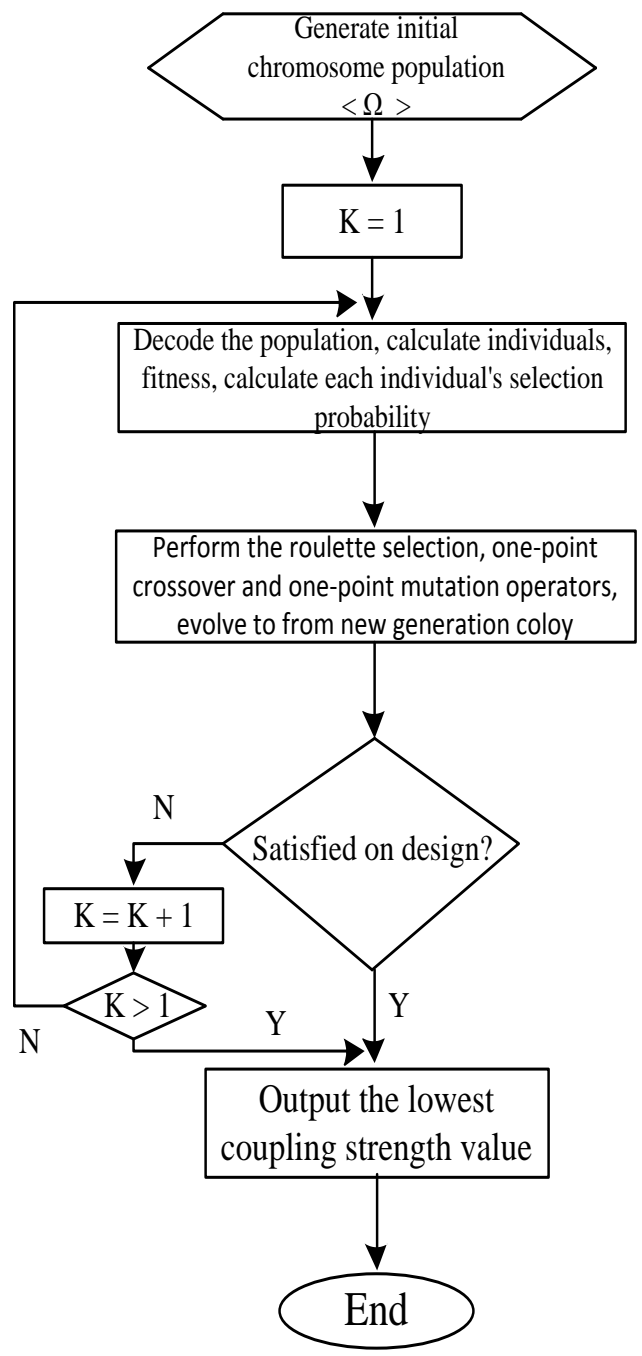

Figure 2: Genetic algorithm flowchart

Moreover, in Fig. 3, the dispersion responses range from approximately zero ps/nm (i.e. at the minimum value of grating length (i.e. $L_{g} \leq 2 \mathrm{~mm}$ )) to several thousand $\mathrm{ps} / \mathrm{nm}$ at high grating length (i.e $L_{g} \geq 30 \mathrm{~mm}$ ) based on the value of $\xi$. However, as it is well known the minimum value of grating length leads to reduce the effective reflectivity for the gratings fiber and then the performance of the system [2, 11]. Therefore, we are forced to work with high grating length to ensure high reflectivity operating. So, this leads to increase the dispersion based on the $\xi$ value. Also, the results obtained by the numerical analysis show that by increasing the $\xi$ value from $2.5 / \mathrm{m}$ to $60 / \mathrm{m}$, the dispersion responses are reduced significantly with $L_{g}$ and these results are totally consistent with that calculated by the genetic algorithms. This is due to increase the coupling strength between the forward and backward waves which leads to increase the gratings reflectivity and then reduces the dispersion value $[11,14]$.

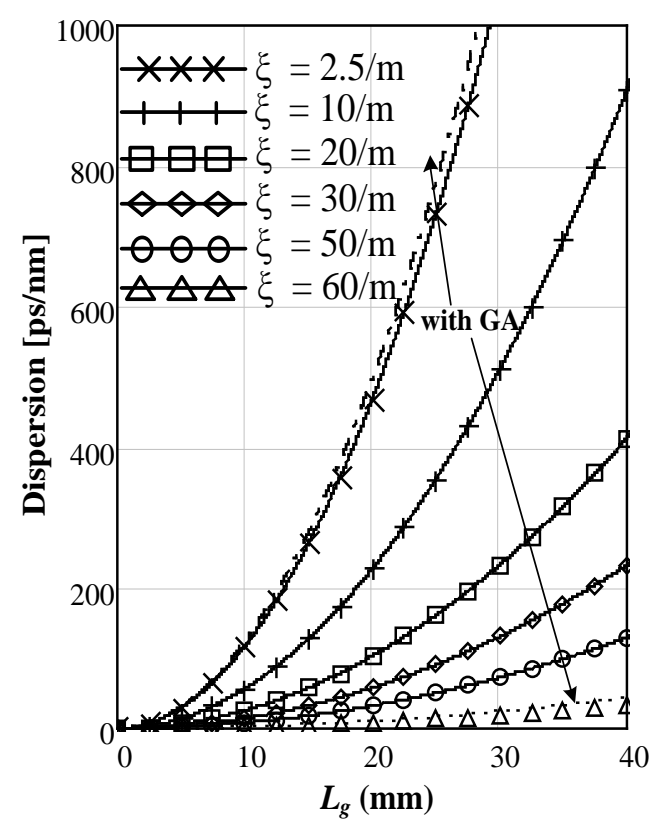

Figure 3: Dispersion properties of uniform POF function to gratings length $\left(L_{g}\right)$ at different $\xi$ value.

One of important parameters that controls the reflectivity and the spectral shape of the fiber grating is the grating's average refractive index change $\left(\delta_{n}\right)$ where, it may cause shift in the center wavelength [11]. Assuming that the $\delta$ distributes uniformly across the fiber core and is nonexistent in the outside region, which is often true due to the lack of photosensitivity of the cladding.

Figures 4 and 5 show numerically the dispersion responses based on the optimal values for the $\xi$ that are calculated by the genetic algorithms at different $\delta n$ values. From Figs 4 and 5 , it is obvious that the dispersion responses are affected by the value of $\delta_{n}$. This can be understood as: by reducing $\delta_{n}$ to a small value $\left(\delta_{n}\right.$ $\rightarrow 0$ ), the wavelength is shifted to a region near their Bragg condition, which results in increment in the grating reflectivity, leads a reduction in the dispersion value $[11,14]$. It is the very important that at the same $\xi$ value, the dispersion amplitude in Fig. 4 is less than that of Fig. 5.

Finally, Temperature is one of the most important factors that affects system performance, where its impact on the model parameters cannot 
be predict. Therefore, investigating its effect on the dispersion response is very important. Figure 6 is shows the effect of temperature $(T)$ variation on the dispersion properties. Results in Fig. 6 (a) and (b) are obtained numerically based on the optimal values for the $\xi$ that are calculated by the genetic algorithms. In this analysis, $T$ has varied from $T_{o}\left(T_{o}\right.$ is assumed as a reference temperature (i.e. $\left.T_{o}=25{ }^{\circ} \mathrm{C}\right)$ ) to $3 T_{o}$. As shown, the change in dispersion response with temperature in Fig. 6(a) is more linear than that in (b), while the value in (b) is less than that in (a). This change is basically due to the temperature dependence for the refractive index of the fiber grating [2, 11, 14] which by shifting temperature from its reference value, the refractive index is changed leads to a shift in the Bragg condition to another wavelength. However, at the high $\xi$ value calculated by the GA, the dispersion properties with temperature variation are less than that at the low $\xi$. where, with the increase of the $\xi$, the coupling between the forward and backward waves increases [11], then the wavelength shift is decreases remarkably and therefore the increase of the $\xi$ help to decrease the temperature effect.
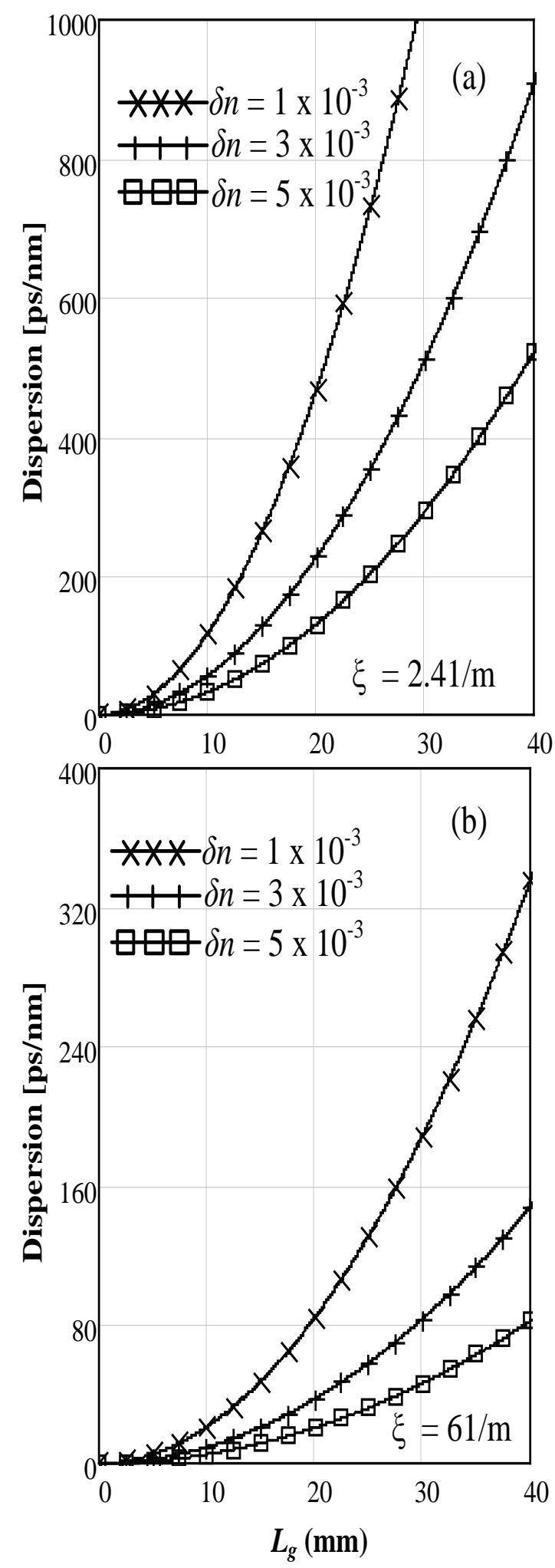

Figure 4: Effect of average refractive index $(\delta n)$ on dispersion properties of uniform POF function to gratings length $\left(L_{g}\right)$ for (a) $\xi=2.41 / \mathrm{m}$ and, (b) $\xi=$ $61 / \mathrm{m}$. 

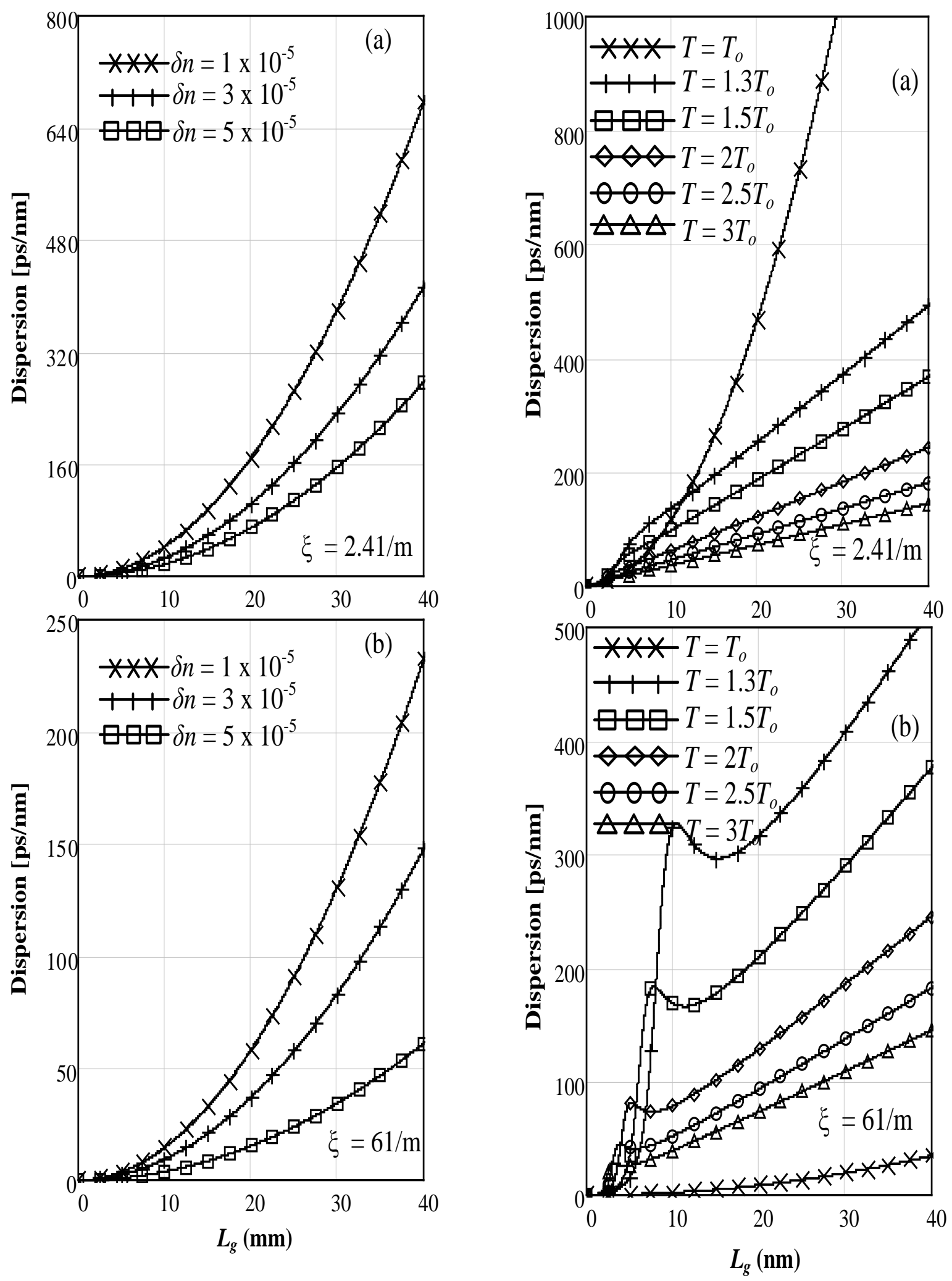

Figure 5: Effect of average refractive index $(\delta n)$ on dispersion properties of uniform POF function to gratings length $\left(L_{g}\right)$ for (a) $\xi=2.41 / \mathrm{m}$ and, (b) $\xi=$ $61 / \mathrm{m}$.

Figure 6: Effect of temperature (T) variation on dispersion properties of uniform POF function to gratings length $\left(L_{g}\right)$ (a) $\xi=2.41 / \mathrm{m}$ and (b) $\xi=61 / \mathrm{m}$. 


\section{Conclusion}

In this study, we propose a method of dispersion optimization for the POF Bragg gratings. Effects of the coupling strength $(\xi)$ factor, average refractive index $(\delta n)$ and temperature $(T)$ change on the dispersion properties have been analyzed numerically. Results showed that by optimizing $\xi$ and reducing $\delta$, the dispersion properties have reduced significantly. Also, results showed that the effect of temperature can be minimized by operating at the high $\xi$ value. These results are very promising for the dispersion reduction in WDM systems.

\section{References}

[1] Hisham K. Hisham, Ahmed F. Abas, Ghafour A. Mahdiraji, Mohammd A. Mahdi and Ahmed S. Muhammad Noor, "Relative Intensity Noise Reduction by Optimizing Fiber Grating Fabry-Perot Laser Parameters', IEEE J. Quantum Electron., vol. 48, pp. 385-393, 2012.

[2] Hisham K. Hisham, "Numerical Analysis of Thermal Dependence of the Spectral Response of Polymer Optical Fiber Bragg Gratings", Iraq J. Electrical and Electronic Engineering, Vol.12, pp.85-95, 2016.

[3] L. Eldada, L. W. Shacklette, “Advances in polymer integrated optics,” IEEE J. Sel. Top. Quantum Electron., vol. 6, pp. 54-68, 2000.

[4] H. Y. Liu, G. D. Peng, P. L. Chu, "Thermal stability of gratings in PMMA and CYTOP polymer fibers,” Opt. Commun., vol. 204, pp. 151-156, 2002.

[5] G. Jeong, J. -H. Lee, M. Y. Park, C. Y. Kim, S. -H. Che, W. Lee, B. W. Kim, “Over 26$\mathrm{nm}$ wavelength tunable external cavity laser based on polymer waveguide platforms for WDM access networks," IEEE photon Tech.Lett., vol. 18, pp. 2102-2104, 2006.

[6] Y. -O. Noh, H. -J. Lee, J. J. Ju, M. -S. Kim, S. H. Oh, M. -C. Oh, "Continuously tunable compact lasers based on thermo-optic polymer waveguides with Bragg gratings,” Opt. Express, vol. 16, pp. 18194-18201, 2008.

[7] T. Imai, T. Komukai and M. Nakazawa, "Dispersion Tuning of a Linearly Chirped Fiber Bragg Grating Without a Center Wavelength Shift by Applying a Strain Gradient”, IEEE Photonic. Technolog Lett., vol. 10, pp. 845-847, 1998.

[8] G. Yabre, "Theoretical Investigation on the Dispersion of Graded-Index Polymer Optical Fibers”, J. Lightwave Technol., vol. 18, pp. 869877, 2000.

[9] Y. Xie,S. Lee, Z. Pan, J.-X. Cai, A. E. Willner,V. Grubsky, D. S. Starodubov, E. Salik, and J. Feinberg, "Tunable Compensation of the Dispersion Slope Mismatch in DispersionManaged Systems Using a Sampled Nonlinearly Chirped FBG”, IEEE Photonic. Technolog Lett., vol. 1417-419, 2000.

[10] Z. Pan, Y. W. Song, C. Yu, Y. Wang, Q. Yu, J. Popelek, H. Li, Y. Li, and Alan Eli Willner, "Tunable Chromatic Dispersion Compensation in 40-Gb/s Systems Using Nonlinearly Chirped Fiber Bragg Gratings”, J. Lightwave Technol., vol.20, pp. 2239-2246, 2002.

[11] A. O. K. Kalli, "Fiber Bragg GratingFundamentals and Applications in Telecommunications and Sensing," Artech House, Boston, 1999.

[12] D. DHAWAN and N. GUPTA, "OPTIMIZATION OF FIBER BASED DISPERSION COMPENSATION IN RZ AND NRZ DATA MODULATION FORMATS”, J. Engineering Science and Technology, Vol. 6, pp. 651 - 663, 2011.

[13]G. Kaur and G. Kaur, "Mitigation of Chromatic Dispersion using Different Compensation Methods in Optical Fiber Communication: A Review”, Int. J. of Engineering and Management Research, vol. 4, pp. 21-27, 2014.

[14]M. G. Kuzyk,’Polymer optical fiber: Materials, Physics, and Applications”, by Taylor \& Francis Group, 2007.

\section{أداء ألالياف الضوئية البلاستيكية المحززة بتشتت منخفض بأستخدام الخوارزميات الوراثية

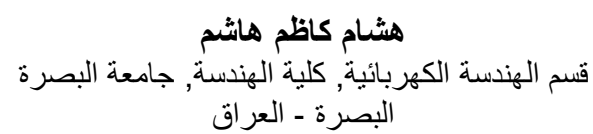

ألخلاصة

في هذا البحث, نقترح طريقة لتقليل التشتت في ألياف براغ الضونئية البلاستيكية المحززة (POF) أعنمادا على أيجاد القيمة المثلى

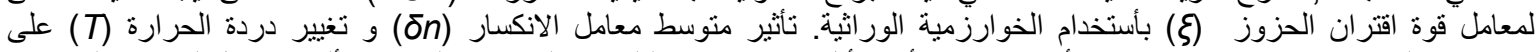

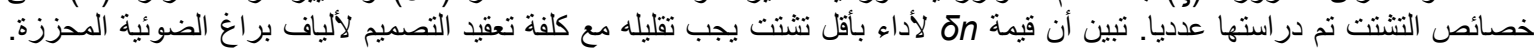

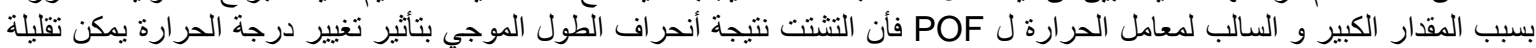

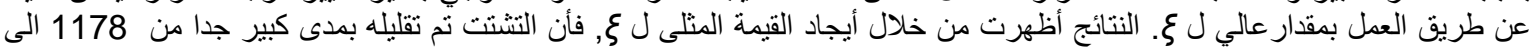
11.5 بيكو ثنانية/نانو متر لحزوز بطول بطول 30 مليمنر. 